\title{
Applications of some basic theorems in the KKM theory
}

Sehie Park ${ }^{1,2}$

Correspondence: shpark@math.snu. ac.kr

${ }^{1}$ The National Academy of Sciences, Republic of Korea, Seoul 137-044, Korea

Full list of author information is available at the end of the article

\section{Abstract}

In this review, we introduce a new KKM-type theorem for intersectionally closedvalued KKM map on abstract convex spaces and its direct consequences such as a Fan-Browder-type fixed point theorem and maximal element theorems. For these basic theorems of the KKM theory, we review previously obtained particular consequences of them mainly due to the author and their recent applications obtained by other authors. Therefore, those applications might be improved by following our new results.

2010 Mathematics Subject Classification: 47H04; 47H10; 52A99; 54H25; 55M20; 58E35; 90D13.

Keywords: intersectionally closed, unionly open, abstract convex space, KKM-type theorem, fixed point, maximal element

\section{Introduction}

The KKM theory, first called by the author [1], is the study on applications of equivalent formulations of the KKM theorem due to Knaster, Kuratowski, and Mazurkiewicz. The KKM theorem provides the foundations for many of the modern essential results in diverse areas of mathematical sciences.

Some of the basic theorems which are useful to applications of the KKM theory were first obtained by Ky Fan, Browder, and others for convex subsets of topological vector spaces (not necessarily Hausdorff). Later extensions of the theory were due to Lassonde for convex spaces, Horvath for $H$-spaces, Park for $G$-convex spaces, and others; see [2] and the references therein.

Recently, the KKM theory is extended to abstract convex spaces by the author and we obtained new results in such frame; see [3] and the references therein. In fact, there are very large numbers of equivalent formulations, generalizations, and applications of the KKM theorem. On the other hand, Luc et al. [4] introduced a meaningful concept of intersectionally closed-valued multimaps and applied them to several related problems.

In this review, we introduce the new KKM-type theorem for intersectionally closedvalued KKM maps and its simple consequences such as a Fan-Browder-type fixed point theorem and maximal element theorems. We also recall some previously obtained particular results of them mainly due to the author and their recent applications obtained by many other authors. Therefore, these applications might be improved by following our new results.

(c) 2011 Park; licensee Springer. This is an Open Access article distributed under the terms of the Creative Commons Attribution License (http://creativecommons.org/licenses/by/2.0), which permits unrestricted use, distribution, and reproduction in any medium, provided the original work is properly cited. 
In Section 2, we introduce basic concepts on our abstract convex spaces and a basic KKM theorem by following [3]. We give also the new concepts of intersectionally closed and unionly open sets due to Luc et al. [4]. Section 3 devotes to deduce a new KKM theorem, a Fan-Browder fixed point theorem, and maximal element theorems. In Section 4, we give some known particular KKM-type theorems and indicate some of their known applications. In Section 5, we give some known Fan-Browder-type fixed point theorems mainly due to the author and indicate their known applications by other authors. Section 6 devotes to give some known particular maximal element theorems and to indicate their known applications. Finally, in Section 7, we introduce several applications of related fixed point theorems due to the author.

\section{Abstract convex spaces}

In this section, we follow [3].

A multimap or simply a map $F: X \rightarrow Y$ is a function $F: X \rightarrow 2^{Y}$ to the power set of $Y$ and $F^{-}: Y \rightarrow X$ is defined by $F^{-}(y):=\{x \in X \mid y \in F(x)\}$ for $y \in Y$. Let $\langle D\rangle$ denote the set of all nonempty finite subsets of a set $D$.

Definition 2.1. An abstract convex space $(E, D ; \Gamma)$ consists of a topological space $E$, a nonempty set $D$, and a multimap $\Gamma:\langle D\rangle \rightarrow E$ with nonempty values $\Gamma_{A}:=\Gamma(A)$ for $A \in$ $\langle D\rangle$.

For any nonempty $D^{\prime} \subset D$, the $\Gamma$-convex hull of $D^{\prime}$ is denoted and defined by

$$
\operatorname{co}_{\Gamma} D^{\prime}:=\bigcup\left\{\Gamma_{A} \mid A \in\left\langle D^{\prime}\right\rangle\right\} \subset E .
$$

A subset $X$ of $E$ is called a $\Gamma$-convex subset of $(E, D ; \Gamma)$ relative to $D^{\prime}$ if for any $N \in$ $\left\langle D^{\prime}\right\rangle$, we have $\Gamma_{N} \subset X$, that is, $\operatorname{co}_{\Gamma} D^{\prime} \subset X$.

When $D \subset E$ in $(E, D$; $)$, a subset $X$ of $E$ is said to be $\Gamma$-convex if $\operatorname{co}_{\Gamma}(X \cap D) \subset X$; in other words, $X$ is $\Gamma$-convex relative to $D^{\prime}:=X \cap D$. In case $E=D$, let $(E ; \Gamma):=(E, E ; \Gamma)$.

If $E$ is compact, then $(E, D ; \Gamma)$ is called a compact abstract convex space.

Example 2.2. Many known examples of abstract convex spaces are given in [3] and the references therein.

Definition 2.3. Let $(E, D ; \Gamma)$ be an abstract convex space. If a map $G: D \multimap E$ satisfies

$$
\Gamma_{A} \subset G(A):=\bigcup_{\gamma \in A} G(\gamma) \quad \text { for all } A \in\langle D\rangle,
$$

then $G$ is called a KKM map.

We have abstract convex subspaces as the following simple observation shows:

Proposition 2.4. [5] For an abstract convex space $(E, D ; \Gamma)$ and a nonempty subset D' of $D$, let $X$ be a $\Gamma$-convex subset of E relative to $D^{\prime}$ and $\Gamma^{\prime}:\left\langle D^{\prime}\right\rangle \rightarrow X$ a map defined by

$$
\Gamma_{A}^{\prime}:=\Gamma_{A} \subset X \text { for } A \in\left\langle D^{\prime}\right\rangle .
$$

Then, (X, D'; $\Gamma$ ') itself is an abstract convex space called a subspace relative to $D^{\prime}$.

Definition 2.5. The partial KKM principle for an abstract convex space $(E, D ; \Gamma)$ is the statement that, for any closed-valued KKM map $G: D \multimap E$, the family $\{G(z)\}_{z \in D}$ has the finite intersection property. The KKM principle is the statement that the same property also holds for any open-valued KKM map.

An abstract convex space is called a KKM space if it satisfies the KKM principle. 
Example 2.6. Many known examples of KKM spaces are given in [3] and the references therein.

From the partial KKM principle, we have the following KKM-type theorem which is a particular form of [[6], Theorem C] and corrects [[3], Theorem 3]:

Theorem 2.7. Let $(E, D ; \Gamma)$ be an abstract convex space satisfying the partial KKM principle and $G: D \rightarrow E$ a map such that

(1) $\bar{G}$ is a KKM map, and

(2) there exists a nonempty compact subset $K$ of $E$ such that either

(i) $\bigcap_{z \in M} \overline{G(z)} \subset$ Kfor some $M \in\langle D\rangle$; or

(ii) for each $N \in\langle D\rangle$, there exists a compact $\Gamma$-convex subset $L_{N}$ of $E$ relative to some $D^{\prime} \subset D$ such that $N \subset D^{\prime}$ and

$$
\overline{L_{N}} \cap \bigcap_{z \in D^{\prime}} \overline{G(z)} \subset K .
$$

Then $K \cap \bigcap_{z \in D} \overline{G(z)} \neq \emptyset$.

Recall that the main conclusions of most of KKM-type theorems follow from the form

$$
\bigcap_{z \in D} \overline{G(z)} \neq \varnothing
$$

for a multimap $G: D \multimap E$.

Consider the following related four conditions:

(a) $\bigcap_{z \in D} \overline{G(z)} \neq \emptyset$ implies $\bigcap_{z \in D} G(z) \neq \emptyset$.

(b) $\bigcap_{z \in D} \overline{G(z)}=\overline{\bigcap_{z \in D} G(z)}$ ( $G$ is intersectionally closed-valued [4]).

(c) $\bigcap_{z \in D} \overline{G(z)}=\bigcap_{z \in D} G(z)$ ( $G$ is transfer closed-valued).

(d) $G$ is closed-valued.

The authors of [4] noted that (a) $\Leftarrow$ (b) $\Leftarrow(\mathrm{c}) \Leftarrow$ (d) and gave some examples of multimaps satisfying (b) but not (c). Therefore, it would be better to deal with condition (b) instead of (c) in the KKM theory.

Example 2.8. The following maps $G$ are intersectionally closed-valued, but not transfer closed-valued:

(1) $G(z)=(0,1)$ for every $z \in[0,1]$ is a constant multimap from $D=[0,1]$ to $E=$ $[0,1]$; see $[4]$.

(2) $G(z)$ is a convex set in a Euclidean space having a relative interior point in common; see Rockafellar [[7], Theorem 6.5].

(3) For a given subset $E$ of a topological vector space with $x^{*} \in E$, each $G(z), z \in D$, is a nicely star-shaped at $x^{*}$; see [4].

For a multimap $G: D \multimap E$, consider the following related four conditions: 
(a) $\bigcup_{z \in D} G(z)=E$ implies $\bigcup_{z \in D}$ Int $G(z)=E$.

(b) Int $\bigcup_{z \in D} G(z)=\bigcup_{z \in D}$ Int $G(z)$ ( $G$ is unionly open-valued [4]).

(c) $\bigcup_{z \in D} G(z)=\bigcup_{z \in D}$ Int $G(z)$ (G is transfer open-valued).

(d) $G$ is open-valued.

Proposition 2.9. [4] The multimap $G$ is intersectionally closed-valued (resp., transfer closed-valued) if and only if its complement $G^{c}$ is unionly open-valued (resp., transfer open-valued).

In view of this proposition, there are proper examples of unionly open-valued maps.

\section{A KKM theorem, a fixed point theorem, and maximal element theorems}

From the partial KKM principle we have the following KKM-type theorem:

Theorem 3.1. Under the hypothesis of Theorem 2.7, the following hold:

( $\alpha$ ) If $G$ is transfer closed-valued, then $K \cap \bigcap_{z \in D} G(z) \neq \emptyset$.

( $\beta)$ If $G$ is intersectionally closed-valued, then $\bigcap_{z \in D} G(z) \neq \emptyset$.

Proof. Since $\bar{G}$ is a KKM map with closed values, by Theorem 2.7, we have $K \cap \bigcap_{z \in D} \overline{G(z)} \neq \emptyset$.

$(\alpha)$ Since $G$ is transfer closed-valued, we have

$$
\bigcap_{z \in D} G(z)=\bigcap_{z \in D} \overline{G(z)} \neq \emptyset .
$$

( $\beta$ ) Since $G$ is intersectionally closed-valued, we have

$$
\overline{\bigcap_{z \in D} G(z)}=\bigcap_{z \in D} \overline{G(z)} \neq \emptyset .
$$

These imply the conclusion.

From the KKM Theorem 3.1, we have a whole intersection property of the Fan-type as follows:

Theorem 3.2. Let $(E, D ; \Gamma)$ be an abstract convex space satisfying the partial KKM principle and $G: D \rightarrow E, H: E \nrightarrow E$ maps satisfying

(2.1) for each $x \in E, x \in H(x)$;

(2.2) for each $x \in E, \mathrm{co}_{\Gamma}\left(D \backslash G^{-}(x)\right) \subset X \backslash H^{-}(x)$; and

(2.3) there exists a nonempty compact subset $K$ of $E$ such that either

(i) $\bigcap_{z \in M} \overline{G(z)} \subset$ Kfor some $M \in\langle D\rangle$; or

(ii) for each $N \in\langle D\rangle$, there exists a compact $\Gamma$-convex subset $L_{N}$ of $E$ relative to some $D^{\prime} \subset D$ such that $N \subset D^{\prime}$ and

$\overline{L_{N}} \cap \bigcap_{z \in D^{\prime}} \overline{G(z)} \subset K$.

( $\alpha$ ) If $G$ is transfer closed-valued, then $K \cap \bigcap_{z \in D} G(z) \neq \emptyset$. 
( $\beta$ ) If $G$ is intersectionally closed-valued, then $\bigcap_{z \in D} G(z) \neq \emptyset$.

Proof. In view of Theorem 3.1, it suffices to show that (2.1) and (2.2) imply that $G$ is a KKM map. Suppose that there exists an $N \in\langle D\rangle$ such that $\Gamma_{N} \notin G(N)$; that is, there exist an $x \in \Gamma_{N}$ such that $x=\notin G(y)$ for all $y \in N$. In other words, $N \in\left\langle D \backslash G^{-}(x)\right\rangle$. By (2.2), $\Gamma_{N} \subset X \backslash H^{-}(x)$. Since $x \in \Gamma_{N}$, we have $x \notin H^{-}(x)$ or $x \notin H(x)$. This contradicts (2.1).

From Theorem 3.2, we have the following fixed point theorem:

Theorem 3.3. Let $(E, D ; \Gamma)$ be an abstract convex space satisfying the partial KKM principle, and $S: D \rightarrow E, T: E \rightarrow E$ maps. Suppose that

(3.1) for each $x \in E, \mathrm{Co}_{\Gamma} S^{-}(x) \subset T^{-}(x)$;

(3.2) $E=S(D)$; and

(3.3) there exists a nonempty compact subset $K$ of $E$ such that either

(i) $\bigcap_{z \in M} \overline{E \backslash S(z)} \subset K$ for some $M \in\langle D\rangle$; or

(ii) for each $N \in\langle D\rangle$, there exists a compact $\Gamma$-convex subset $L_{N}$ of $E$ relative to some $D^{\prime} \subset D$ such that $N \subset D^{\prime}$ and

$$
\overline{L_{N}} \cap \bigcap_{z \in D^{\prime}} \overline{E \backslash S(z)} \subset K .
$$

( $\alpha$ ) If $S$ is transfer open-valued, then there exists an $\bar{x} \in K$ such that $\bar{x} \in T(\bar{x})$.

$(\beta)$ If $S$ is unionly open-valued, then there exists an $\bar{x} \in E$ such that $\bar{x} \in T(\bar{x})$.

Proof. Suppose that $x \notin T(x)$ for all $x \in E$. Let $G(z):=E \backslash S(z)$ for $z \in D$ and $H(x):=E$ $\backslash T(x)$ for $x \in E$. Then all of the requirements of Theorem 3.2 are satisfied.

$(\alpha)$ Therefore, by Theorem 3.2, there exists a $y_{0} \in K \cap \bigcap_{z \in D} G(z)$; that is, $y_{0} \in K$ such that $y_{0} \notin S(z)$ for all $z \in D$. This contradicts (3.2).

( $\beta$ ) Similar.

From Theorem 3.3, we have the following maximal element theorem:

Theorem 3.4. Let $(E, D ; \Gamma)$ be an abstract convex space satisfying the partial KKM principle, and $S: E \rightarrow D, T: E \rightarrow E$ maps. Suppose that

(4.1) for each $x \in E, \operatorname{co}_{\Gamma} S(x) \subset T(x)$;

(4.2) for each $x \in E, x \notin T(x)$; and

(4.3) there exists a nonempty compact subset $K$ of $E$ such that either

(i) $\bigcap_{z \in M} \overline{E \backslash s-(z)} \subset K$ for some $M \in\langle D\rangle$; or

(ii) for each $N \in\langle D\rangle$, there exists a compact $\Gamma$-convex subset $L_{N}$ of $E$ relative to some $D^{\prime} \subset D$ such that $N \subset D^{\prime}$ and

$\overline{L_{N}} \cap \bigcap_{z \in D^{\prime}} \overline{E \backslash S^{-}(z)} \subset K$. 
( $\alpha$ ) If $S$ is transfer open-valued, then there exists a maximal element $\bar{x} \in K$, that is, $S(\bar{x})=\emptyset$.

$(\beta)$ If $S$ is unionly open-valued, then there exists a maximal element $\bar{x} \in E$, that is, $S(\bar{x})=\emptyset$.

Proof. Replace $S$ and $T$ in Theorem 3.3 by $S^{-}$and $T^{-}$, respectively.

From Theorem 3.1, we can deduce another maximal element theorem:

Theorem 3.5. Let $(E, D ; \Gamma)$ be an abstract convex space satisfying the partial KKM principle, and $S: D \rightarrow E$ a map. Suppose that

(5.1) for each $A \in\langle D\rangle$ and each $y \in \Gamma_{A}$, there exists $z \in A$ such that $y \notin S(z)$.

(5.2) Suppose that there exists a nonempty compact subset $K$ of $E$ such that either

(i) $\bigcap_{z \in M} \overline{E / S(z)} \subset K$ for some $M \in\langle D\rangle$; or

(ii) for each $N \in\langle D\rangle$, there exists a compact $\Gamma$-convex subset $L_{N}$ of $E$ relative to some $D^{\prime} \subset D$ such that $N \subset D^{\prime}$ and

$\overline{L_{N}} \cap \bigcap_{z \in D^{\prime}} \overline{E \backslash S(z)} \subset K$.

$(\alpha)$ If $S$ is transfer open-valued, then $S^{-}$has a maximal element $\bar{x} \in K$, that is, $S^{-}(\bar{x})=\emptyset$.

$(\beta)$ If $S$ is unionly open-valued, then $S^{-}$has a maximal element $\bar{x} \in E$, that is, $S^{-}(\bar{x})=\emptyset$.

Proof. Let $G(z):=E \backslash S(z)$ for each $z \in D$. For any $A \in\langle D\rangle$ and any $y \in \Gamma_{A}$, by (5.1), there exists $z \in A$ such that $y \notin S(z)$, that is, $y \in G(z)$. Then $G: D \multimap E$ is a KKM map. Note that $G$ satisfies all requirements of Theorem 3.1. Hence, there exists $\bar{x} \in K$ for the case $(\alpha)$ [resp., $\bar{x} \in E$ for $(\beta)$ ] such that $\bar{x} \in \bigcap_{z \in D} G(z)$. Hence $\bar{x} \notin S(z)$ for all $z \in$ $D$. This shows $S^{-}(\bar{x})=\emptyset$.

In Theorems 2.7 and 3.1-3.5, for the $\Gamma$-convex subset $L_{N}$ of $E$, its closure $\overline{L_{N}}$ can be replaced by simply $L_{N}$ whenever one of the following holds; see [8].

(1) $E$ is Hausdorff (hence, every compact subset is closed).

(2) $K$ is closed.

(3) $L_{N}$ itself is closed.

\section{Particular KKM theorems and applications}

There are several hundreds of articles on generalizations of the KKM theorem and their applications. In a recent study by Park [6], we recalled a number of KKM-type theorems due to the author in order to give a short history on such theorems with related studies.

In this section, we introduce some of simple consequences of Theorem 3.1 and only a few applications (I)-(IV) obtained by other authors. We will omit the proofs.

(I) In 2000, Song [9] deduced the following:

Proposition 4.1. [9]Let $E$ be a topological vector space, $X$ a nonempty convex subset of $E, D$ a nonempty subset of $X$, and $K$ a nonempty compact subset of $X$. Let $G: D \rightarrow X$ 
be a multimap such that

(1) for each $x \in D$ and for each nonempty compact subset $B$ of $X$, the set $G(x) \cap B$ is closed in $B$

(2) for each finite subset $N$ of $D$, co $N \subset G(N)$;

(3) for each finite subset $N$ of $D$, there exists a nonempty compact convex subset $L_{N}$ of $X$ containing $N$ such that, $\forall y \in L_{N} \mid K, \exists x \in L_{N} \cap D$ with $y \notin G(x)$.

Then $\exists \hat{y} \in K$ such that $\hat{y} \in G(x) \forall x \in D$.

This is a particular form of [[1], Theorem 3 or 4']. Note that $G(x)$ can be merely closed instead of the "compactly" closedness in (1) since the topology of $X$ can be replaced by its compactly generated extension; see [10].

In our previous study [3], we showed that the partial KKM property implies various forms of the Fan-type minimax inequality and variational inequalities.

Similarly, Song [9] deduced from Proposition 4.1 a vector and set-valued generalization of the Fan minimax inequality. This is applied to several existence theorems for generalized vector variational inequalities involving certain set-valued operators. Moreover, he gave a certain relationship between a kind of generalized vector variational inequality (VVI) and a vector optimization problem.

(II) In 2000, Song [11] obtained the following:

Proposition 4.2. [11]Let $X$ be a nonempty convex subset of a real topological vector space $E, D \subset X$ nonempty, and $K \subset X$ nonempty compact. Let $G: D \rightarrow X$ be a map such that

(1) $G$ is transfer compactly closed-valued on $D$;

(2) for every compact convex subset $X_{0}$ of $X$ and for each $N \in\left\langle D \cap X_{0}\right\rangle$, $\operatorname{co}(N) \subset \bigcup_{y \in N} c 1_{X_{0}}\left(G(y) \cap X_{0}\right)$;

(3) for each $N \in\langle D\rangle$, there exists a nonempty compact convex subset $L_{N}$ of $X$ containing $N$ such that

$$
\bigcap_{\gamma \in L_{N} \cap D} \mathrm{c1} 1_{L_{N}}\left(G(y) \cap L_{N}\right) \subset K .
$$

Then $K \cap\left(\bigcap_{y \in A} G(y)\right) \neq \emptyset$.

This is particular to Theorem 2.7 as we can eliminate "compactly" in (1).

In [11], by applying Proposition 4.2, an existence result for a generalized vector equilibrium problem was obtained. This was used to obtain existence results for vector equilibrium problems and vector variational inequalities. Its author adopted artificial and impractical concepts like compactly closed and compact closure, which can be eliminated by adopting the compactly generated extension of the original topology.

Note that $(X, D)$ in Propositions 4.1 and 4.2 is a convex space in the sense of Park.

(III) A modification of Proposition 4.2 is given in [12] and applied to similar problems.

(IV) A simple G-convex space version of the Fan KKM lemma or Theorem 2.7 is as follows: 
Proposition 4.3. Let $(X, D ; \Gamma)$ be a $G$-convex space and $G: D \rightarrow X$ a closed-valued $K K M$ map. If $G(x)$ is compact for some $x \in D$, then $\bigcap_{x \in D} G(x) \neq \emptyset$.

There are a large number of applications of the Fan KKM lemma and it is hard to list all of them. Recently its G-convex space versions like Proposition 4.3 also were shown to have applications. For example, Mitrović $[13,14]$ published some applications of Proposition 4.3 to various problems in G-convex spaces as follows.

In 2006 [13], he proved a best approximation theorem and applied it to the existence of maximal elements and coincidence points.

In 2007 [14], he proved the existence of a solution to the scalar equilibrium problem and applied it to best approximations and simultaneous approximations.

\section{Particular fixed point theorems and applications}

In this section, we introduce simple consequences of Theorem 3.3 and their applications (I)-(XIII) obtained by other authors. We will omit the proofs.

Proposition 5.1. Let $x$ be a nonempty convex subset of a topological vector space $E$, $A, B: X \multimap X$ two multimaps, and $K$ a nonempty compact subset of $X$. Suppose that

(1) for each $x \in X, A(x) \subset B(x)$ and $B(x)$ is convex;

(2) for each $x \in K, A(x) \neq \varnothing$;

(3) for each $y \in X, A^{-}(y)$ is open in $X$;

(4) for each finite subset $N$ of $X$, there exists a compact convex subset $L_{N}$ of $X$ containing $N$ such that $A(x) \cap L_{N} \neq \varnothing$ for all $x \in L_{N} \mid K$.

Then $B$ has a fixed point.

This is originally given as a particular form of [[1], Theorem 1] or [[15], Theorem 5].

In order to prove some existence results for noncompact settings, many authors applied Proposition 5.1 as follows:

(I) In 1998, Lee and Kum [16] applied Proposition 5.1 to obtain two theorems on the existence of solutions of VVI problems with a noncompact setting in a Hausdorff topological vector space. The results generalize previous studies of Lai and Yao, Yu and Yao. In addition, the equivalent relation between solutions of generalized Minty vector variational inequality and generalized vector-minimum points of vector optimization problems was shown.

(II) In 1998, Park and Kum [17] applied Proposition 5.1 with $A=B$ to a general existence theorem on a generalized variational inequalities and some of its consequences.

(III) In 2000, Lee and Kum [18] applied Proposition 5.1 to several existence theorems of solutions of implicit vector variational inequalities for multimaps with or without generalized pseudomonotonicity. These results extend and unify corresponding earlier studies.

(IV) In 2001, Ansari et al. [19] obtained some existence results for solutions to the generalized vector equilibrium problem for $C_{x}$-pseudomonotone as well as $C_{x}$-quasimonotone multimaps by applying Proposition 5.1. They also proved an existence result without any kind of monotonicity assumption. As special cases, they derived existence results for solutions to the implicit vector variational inequalities.

(V) In 2002, Kum and Lee [20] applied Proposition 5.1 to some existence of solutions of the implicit vector variational inequality problem (IVVI) for noncompact- 
valued multimaps. This IVVI contains various vector variational-like inequalities and vector equilibrium problems as particular cases. They gave several existence results of solutions for IVVI, thus extending similar results from their previous article [18], and they established such existence results under additional generalized hemicontinuity and pseudomonotonicity type assumptions. Some other existence results, based on a selection theorem, were also presented.

(VI) In 2002, Lin and Cheng [21] applied Proposition 5.1 to the existence results of two types of equilibrium problems-the constrained or the competitive Nash type equilibrium problems with multivalued payoff functions. In these two problems, the authors found a strategy combination such that each player wishes to find a minimal lose from his multivalued payoff function.

(VII) In 2005, Kum and Kim [22] applied Proposition 5.1 to develop the scheme of vector variational inequalities with operator solutions due to Domokos and Kolumbán from the single-valued case into the multi-valued one. They proved the existence of solutions of such inequalities and generalized quasi-vector variational inequalities with operator solutions. Some applications to such inequalities in a normed space were also provided.

(VIII) In 2006, Kum [23] applied the compact case of Proposition 5.1 to further develop the previous study [22] for a more general pseudomonotone operator.

(IX) In 2008, Kum and Kim [24] applied Proposition 5.1 with $A=B$ to new existence theorems on generalized operator equilibrium problem and generalized operator quasiequilibrium problem which includes operator equilibrium problems.

(X) In 2009, Hai et al. [25] established sufficient existence conditions for general quasi-variational inclusion problems, which contain most of variational inclusion problems and quasi-equilibrium problems considered in the literature. The authors made use of Proposition 5.1 and noted that "it turns out that this theorem is suitable to our problem setting and helps to get new results or to sharpen some recent existing ones."

We know that G-convex spaces are typical example of abstract convex spaces. The following extension of the Fan-Browder fixed point theorem to G-convex spaces is well known. For instance, it is a particular case of Theorem 3.3 and [[26], Theorem 3].

Proposition 5.2. Let $(X, D ; \Gamma)$ be a compact G-convex space and $T: X \multimap X, S: X \multimap$ $D$ be two maps satisfying the following conditions:

(i) for each $x \in X, A \in\langle S(x)\rangle$ implies $\Gamma(A) \subset T(x)$;

(ii) $X=\bigcup\left\{\right.$ Int $\left.S^{-}(z) \mid z \in D\right\}$.

Then $T$ has a fixed point.

(XI) In 2006, Balaj [27] applied this to obtain two minimax inequalities in G-convex spaces which extend and improve a large number of generalizations of the Ky Fan minimax inequality and of the von Neumann-Sion minimax principle.

The following consequence of Theorem 3.3 is known in our G-convex space theory:

Proposition 5.3. Let $(X ; \Gamma)$ be a G-convex space and $S, Q: X \rightarrow X$ be two maps satisfying the following conditions:

(i) for each $x \in X, M \in\langle S(x)\rangle$ implies $\Gamma(M) \subset Q(x)$;

(ii) $X=\bigcup_{y \in X} \operatorname{Int} S^{-}(\gamma)$; 
(iii) there exists a nonempty compact subset $K$ of $X$ such that for each $M \in\langle X\rangle$, there exists a compact G-convex subset $L_{M} \subset X$ containing $M$ such that

$$
L_{M} \backslash K \subset \bigcup\left\{\operatorname{Int} S^{-}(y) \mid y \in L_{M}\right\} .
$$

Then there exists $x_{0} \in X$ such that $x_{0} \in Q\left(x_{0}\right)$.

(XII) In 2003, Ding and Park [28] applied this to a class of abstract generalized vector equilibrium problems in G-convex spaces.

(XIII) In 2009, Balaj and Lin [29] claimed that Proposition 5.3 is essentially Theorem 4 of [30] and applied it to obtain a new fixed point theorem for multivalued maps in $G$-convex spaces from which they derived several coincidence theorems and existence theorems for maximal elements. Applications of these results to generalized equilibrium problems and minimax theory were also given.

\section{Particular maximal element theorems and applications}

In this section, we introduce simple consequences of Theorems 3.4 and 3.5, and their applications (I)-(VI) due to other authors. We obtain the following from Theorem 3.4:

Proposition 6.1. [31]Let $(X \supset D ; \Gamma)$ be an H-space and $S: D \rightarrow X, T: X \rightarrow X$ multimaps such that

(1) for each $z \in D, S(z) \subset T(z)$ and $S(z)$ is compactly open; and

(2) for each $y \in X, T(y)$ is $\Gamma$-convex.

Suppose that there exists a nonempty compact subset $K$ of $X$ such that either

(i) $X \backslash K \subset S(M)$ for some $M \in\langle D\rangle$, or

(ii) for each $N \in\langle D\rangle$, there exists a compact $\Gamma$-convex subset $L_{N}$ of $X$ containing $N$ such that

$$
L_{N} \backslash K \subset S\left(L_{N} \cap D\right) .
$$

Then either there exists $y_{0} \in K$ such that $S^{-}\left(y_{0}\right)=\varnothing$ or there exists $x_{0} \in X$ such that $x_{0} \in T\left(x_{0}\right)$.

We indicate some applications of Proposition 6.1 as follows:

(I) The following in 2003 simply follows from Proposition 6.1:

Proposition 6.2. [32] Let $X$ be a nonempty convex subset of a t.v.s. E. Let $S: X \rightarrow X$ be a set-valued mapping satisfying the following conditions:

(i) $x \notin \operatorname{co} S(x)$ and $S^{-}$is open-valued;

(ii) there exist a nonempty compact subset $A$ of $X$ and a nonempty compact convex subset $B$ of $X$ such that for all $x \in X \backslash A$, there exists $z \in B$ such that $x \in \operatorname{Int} S^{-}(z)$.

Then there exists $x \in X$ such that $S(x)=\varnothing$.

(II) In 2004, Guo [33] applied Proposition 6.2 to prove existence theorems of solutions of the complementarity problems for multivalued monotone operator in Banach spaces. 
(III) In 2005, Peng and Yang [34] applied Proposition 6.1 to a new existence result for a multivalued complementarity problem due to Guo [33], which is a correction of a previous result in [33].

The following is a simple consequence of Theorem 3.5:

Proposition 6.3. [35]Let $(X \supset D ; \Gamma)$ be an H-space and $S: D \multimap X, T: X \rightarrow X$ multimaps such that

(1) for each $z \in D, S(z) \subset T(z)$ and $S(z)$ is compactly open; and

(2) for each $A \in\langle D\rangle$ and for each $y \in \Gamma_{A}$, there exists $z \in A$ such that $y \notin T(z)$.

Suppose that there exists a nonempty compact subset $K$ of $X$ such that either

(i) $X \backslash K \subset S(M)$ for some $M \in\langle D\rangle$, or

(ii) for each $N \in\langle D\rangle$, there exists a compact $\Gamma$-convex subspace $L_{N}$ of $X$ containing $N$ such that

$$
L_{N} \backslash K \subset S\left(L_{N} \cap D\right) .
$$

Then either there exists $y_{0} \in K$ such that $S^{-}\left(y_{0}\right)=\varnothing$.

(IV) In 2007, from a KKM-type theorem in [31], Guo and Cho [35] deduced a maximal element theorem as above and applied it to the Browder-Hartmann-Stampacchia variational inequalities and the complementarity problem in Banach spaces and locally convex topological vector spaces, respectively.

(V) In 2010, Xiang et al. [36] studied a class of generalized nonlinear vector variational-like inequality problems, which includes generalized nonlinear VVI problems, generalized VVI problems, generalized vector variational-like inequality problems, and so on. Applying Proposition 6.3, they proved the existence of its solutions in the setting of locally convex t.v.s.

(VI) In 2010, particular forms of Theorems 3.2 and 3.3 for a compact $\Gamma$-convex subspace $X$ were applied to minimax theorems by Yang et al. [37]. In this article, they introduced the concepts of $C$-quasi-concave maps and properly $C$-quasi-concave maps in abstract convex spaces in the sense of Park, where $C$ is a pointed closed convex cone with nonempty interior. By using the Fan-Browder-type fixed point theorem and the maximal element theorem due to Park, they established some minimax theorems for vector-valued mappings in abstract convex spaces. They also give some examples to illustrate their results.

\section{Comments on some related results}

In this section, we are concerned with some other applications of related fixed point theorems other than the preceding basic theorems of the KKM theory.

(I) In 1994, Kum [38] applied the 1992 fixed point theorem for acyclic maps [[1], Theorem 7] to a generalized quasi-variational inequality problem. In a noncompact setting, he presented some general acyclic-valued versions of several known results due to Kim, Shih, and Tan. For further developments, see [39].

(II) In 1996, Lee et al. [40] also applied the 1992 theorem to a vector version of the main result of [36], which is an existence theorem for the vector quasi-variational inequality for multimaps with vector values. 
(III) In 1998, Lin and Park [41] studied some equilibrium problems, quasi-equilibrium problems, and generalized quasi-equilibrium theorems in G-convex spaces. They are based on a selection theorem, a Fan-Browder fixed point theorem (particular to Theorem 3.3), and a KKM-type theorem (particular to Theorem 2.7) on G-convex spaces. Therefore, some results in [41] can be extended to abstract convex spaces satisfying the partial KKM principle.

(IV) In 2002, Kim et al. [42] applied a fixed point theorem on compact Browder maps due to Ben-El-Mechaiekh (see [43]) to an existence result for a VVI involving vector maximal points. Moreover, four kinds of VVIs are shown to be equivalent in the sense that their solution sets are same.

(V) In 2009 [39], we reviewed applications of our fixed point theorems on compact compositions of acyclic maps. Our applications are mainly on acyclic polyhedra, locally convex topological vector spaces, admissible (in the sense of Klee) convex sets, and almost convex or Klee approximable sets in topological vector spaces. Those applications are concerned with general equilibrium problems like as (collective) fixed point theorems, the von Neumann-type intersection theorems, the von Neumann-type minimax theorems, the Nash-type equilibrium theorems, cyclic coincidence theorems, best approximation theorems, (quasi-)variational inequalities, and the Gale-Nikaido-Debreu theorem. Finally, we briefly introduced some related results mainly appeared in other author's studies.

(VI) In [44], let $I$ be a finite or infinite index set, let $X$ be a topological space, and let $\left(Y_{i}, \phi_{N_{i}}\right)_{i \in I}$ be a family of $F C$-spaces. For each $i \in I$, let $A_{i}: X \multimap Y_{i}$ be a set-valued mapping. Some existence theorems of maximal elements for a multimap and a family of multimaps involving a better admissible multimap in the sense of Park are established under noncompact setting of $F C$-spaces. He and Zhang [44] claimed that their results improve and generalize some recent results.

However, the authors adopted artificial and useless concepts on the so-called $F C$ spaces.

(VII) In 2010, Chen and Zhang [45] proved a Fan-Browder-type fixed point theorem under noncompact setting of general topological spaces. By applying the fixed point theorem, several existence theorems of solutions for equilibrium problems are proved under noncompact setting of topological spaces. The authors claimed that these theorems improve and generalize the corresponding results in related literature.

However, the authors of [45] adopted artificial and useless concepts like compact closure, compact interior, transfer compactly open, compactly local intersection property, generalized R-KKM map, etc. due to Ding. Moreover, they claimed that they are concerned with general topological spaces, but their $L_{N}$ is a so-called $F C$-space or, more generally, a G-convex space.

(VIII) Finally, there have appeared a number of fixed point theorems for the multimap classes $\mathfrak{B}$ or $\mathfrak{R C}$ on abstract convex spaces; for example, see Park [46] and the references therein. Moreover, refined versions of the basic theorems in the KKM theory for abstract convex spaces are given in a recent study of Park [47]. 


\section{References}

1. Park, S: Some coincidence theorems on acyclic multifunctions and applications to KKM theory. In: Tan KK (ed.) Fixed Point Theory and Applications. pp. 248-277. World Scientific Publisher, River Edge, NJ (1992)

2. Park, S: Ninety years of the Brouwer fixed point theorem. Vietnam J Math. 27, 187-222 (1997)

3. Park, S: The KKM principle in abstract convex spaces: equivalent formulations and applications. Nonlinear Anal. 73, 1028-1042 (2010). doi:10.1016/j.na.2010.04.029

4. Luc, DT, Sarabi, E, Soubeyran, A: Existence of solutions in variational relation problems without convexity. J Math Anal Appl. 364, 544-555 (2010). doi:10.1016/j.jmaa.2009.10.040

5. Park, S: Elements of the KKM theory on abstract convex spaces. J Korean Math Soc. 45, 1-27 (2008)

6. Park, S: A genesis of general KKM theorems for abstract convex spaces. J Nonlinear Anal Optim. 2(1), 121-132 (2011)

7. Rockafellar, RT: Convex Analysis. Princeton University Press, Princeton, NJ (1970)

8. Park, S: Remarks on certain coercivity in general KKM theorems. Nonlinear Anal Forum. 16, 1-10 (2011)

9. Song, W: Generalized vector variational inequalities. In: Giannessi F (ed.) Vector Variational Inequalities and Vector Equilibria. pp. 381-401. Kluwer Academic Publisher (2000)

10. Park, S: Remarks on some basic concepts in the KKM theory. Nonlinear Anal. 74, 2439-2447 (2011). doi:10.1016/j. na.2010.10.035

11. Song, W: Vector equilibrium problems with set-valued mappings. In: Giannessi F (ed.) Vector Variational Inequalities and Vector Equilibria. pp. 403-421. Kluwer Academic Publisher (2000)

12. Song, W: On generalized vector equilibrium problems. J Comp Appl Math. 146, 167-177 (2002). doi:10.1016/S0377-0427 (02)00426-0

13. Mitrović, ZD: Remarks on best approximations in generalized convex spaces. J Appl Anal. 12, 181-188 (2006) doi:10.1515/JAA.2006.181

14. Mitrović, ZD: On scalar equilibrium problem in generalized convex spaces. J Math Anal Appl. 130, 451-461 (2007)

15. Park, S: Foundations of the KKM theory via coincidences of composites of admissible u.s.c. maps. J Korean Math Soc. 31, 493-519 (1994)

16. Lee, GM, Kum, S: Vector variational inequalities in a Hausdorff topological vector space. Nonlinear Anal Forum. 3, 137-149 (1998)

17. Park, S, Kum, S: An application of Browder-type fixed point theorem to generalized variational inequalities. J Math Anal Appl. 218, 519-526 (1998). doi:10.1006/jmaa.1997.5816

18. Lee, GM, Kum, S: On implicit vector variational inequalities. J Optim Theory Appl. 104, 409-425 (2000). doi:10.1023/ A:1004617914993

19. Ansari, QH, Konnov, IV, Yao, JC: On generalized vector equilibrium problems. Nonlinear Anal. 47, $543-554$ (2001). doi:10.1016/S0362-546X(01)00199-7

20. Kum, S, Lee, GM: Remarks on implicit vector variational inequalities. Taiwan J Math. 6, 369-382 (2002)

21. Lin, L-J, Cheng, SF: Nash-type equilibrium theorems and competitive Nash-type equilibrium theorems. Comp Math Appl. 44, 1360-1378 (2002)

22. Kum, S, Kim, WK: Generalized vector variational and quasi-variational inequalities with operator solutions. J Glob Optim. 32, 581-595 (2005). doi:10.1007/s10898-004-2695-6

23. Kum, S: A variant of the generalized vector variational inequalities with operator solutions. Comm Korean Math Soc. 21, 665-673 (2006)

24. Kum, S, Kim, WK: On generalized operator quasi-equilibrium problems. J Math Anal Appl. 345, 559-565 (2008). doi:10.1016/j.jmaa.2008.04.035

25. Hai, NX, Khanh, PQ, Quan, NH: On the existence of solutions to quasi-variational inclusion problems. J Glob Optim. 45 565-581 (2009). doi:10.1007/s10898-008-9390-y

26. Park, S: Continuous selection theorems in generalized convex spaces. Numer Funct Anal Optim. 20, 567-583 (1999). doi:10.1080/01630569908816911

27. Balaj, M: Two minimax inequalities in G-convex spaces. Appl Math Lett. 19, 235-239 (2006). doi:10.1016/j. aml.2005.06.003

28. Ding, XP, Park, JY: Fixed points of generalized vector equilibrium problems in generalized convex spaces. Indian J Pure Appl Math. 34(6), 973-990 (2003)

29. Balaj, M, Lin, L-J: Fixed points, coincidence points and maximal elements with applications to generalized equilibrium problems and minimax theory. Nonlinear Anal. 70, 393-403 (2009). doi:10.1016/j.na.2007.12.005

30. Yu, Z-T, Lin, L-J: Continuous selection and fixed point theorems. Nonlinear Anal. 52, , 445-455 (2003)

31. Park, S: On minimax inequalities on spaces having certain contractible subsets. Bull Aust Math Soc. 47, 25-40 (1993). doi:10.1017/S0004972700012235

32. Lin, L-J, Yu, Z-T, Ansari, QH, Lai, LP: Fixed point and maximal element theorems with applications to abstract economies and minimax inequalities. J Math Anal Appl. 284, 656-671 (2003). doi:10.1016/S0022-247X(03)00385-8

33. Guo, W: Complementarity problems for multivalued monotone operator in Banach spaces. J Math Anal Appl. 292, 344-350 (2004). doi:10.1016/j.jmaa.2003.11.051

34. Peng, J, Yang, X: On multivalued complementarity problems in Banach spaces. J Math Anal Appl. 307, $245-254$ (2005). doi:10.1016/j.jmaa.2004.10.051

35. Guo, W, Cho, YJ: A new maximal element theorem in H-space with applications. Nonlinear Anal. 68, $2194-2203$ (2008). doi:10.1016/j.na.2007.01.040

36. Xiao, G, Fan, Z, Qi, X: Existence results for generalized nonlinear vector variational-like inequalities with set-valued mapping. Appl Math Lett. 23, 44-47 (2010). doi:10.1016/j.aml.2009.07.023

37. Yang, M-G, Xu, J-P, Huang, N-J, Yu, S-J: Minimax theorems for vector-valued mappings in abstract convex spaces. Taiwan J Math. 14, 719-732 (2010)

38. Kum, SH: A generalization of generalized quasi-variational inequalities. J Math Anal Appl. 182, 158-164 (1994). doi:10.1006/jmaa.1994.1073 
39. Park, S: Applications of fixed point theorems for acyclic maps-a review. Vietnam J Math. 37, 419-441 (2009)

40. Lee, GM, Lee, BS, Chang, S-S: On vector quasi-variational inequalities. J Math Anal Appl. 203, 626-638 (1996). doi:10.1006/jmaa.1996.0401

41. Lin, L-J, Park, S: On some generalized quasi-equilibrium problems. J Math Anal Appl. 224, 167-181 (1998). doi:10.1006/ jmaa.1998.5964

42. Kim, MH, Kum, SH, Lee, GM: Vector variational inequalities involving vector maximal points. J Optim Theory Appl. 114 593-607 (2002). doi:10.1023/A:1016075029509

43. Park, S: Remarks on a fixed point problem of Ben-El-Mechaiekh. Proceedings of the Nonlinear Analysis and Convex Analysis (NACA'98). pp. 79-86.World Scientific Press, Singapore (1999)

44. He, R-H, Zhang, Y: Some maximal elements theorems in FC-spaces. J Inequal Appl (2009). (Article ID 905605), 12. doi:10.1155/2009/905605

45. Chen, Y-A, Zhang, Y-P: A class of Fan-Browder type fixed-point theorem and its applications in topological space. Abst Appl Anal (2010). (Article ID 314616), 9. doi:10.1155/2010/314616

46. Park, S: Fixed point theorems in the new era of the KKM theory. Proceedings of the Fixed Point Theory and Its Applications (ICFPTA-2009). pp. 145-159.Yokohama Publisher (2010)

47. Park, S: New generalizations of basic theorems in the KKM theory. Nonlinear Anal. 74, 3000-3010 (2011). doi:10.1016/j. na.2011.01.020

doi:10.1186/1687-1812-2011-98

Cite this article as: Park: Applications of some basic theorems in the KKM theory. Fixed Point Theory and Applications 2011 2011:98.

\section{Submit your manuscript to a SpringerOpen ${ }^{\circ}$} journal and benefit from:

- Convenient online submission

- Rigorous peer review

- Immediate publication on acceptance

- Open access: articles freely available online

- High visibility within the field

- Retaining the copyright to your article

Submit your next manuscript at $\boldsymbol{s p r i n g e r o p e n . c o m ~}$ 